\title{
Aneurysmal Dilatation of Anterior Cranial Fossa Dural Arteriovenous Fistula: Three Cases
}

\author{
Zheng Liu ${ }^{\mathrm{a}, \mathrm{b}}$, Bangqing Yuan ${ }^{\mathrm{a}}$
}

\begin{abstract}
Aneurysmal dilatation of anterior cranial fossa dural arteriovenous fistula is a rare malformation with a unique and complex angioarchitecture profile. Clinical manifestations, diagnosis and treatment management of three patients with the malformation were retrospectively analyzed. Preoperative examinations revealed all three patients suffered from aneurysmal dilatation; two of them were treated with surgical clipping of the fistula as well as removing of the aneurysm, and cured; one patient died of delayed surgical operation and other complications in two weeks. MRI examination could be used for early diagnosis and cerebral angiography could further confirm the diagnosis. To achieve a favorable outcome for patients the malformation has to be treated as early as possible by surgery or interventional therapy.
\end{abstract}

Keywords: Aneurysmal dilatation; Anterior cranial fossa; Dural arteriovenous fistula

\section{Introduction}

Dural arteriovenous fistula (DAVF) occurs mainly in the vicinity of intracranial venous sinuses and causes abnormal communications between intracranial and extracranial feeding arteries as well as drainage veins, and dural sinuses. It belongs to intracranial vascular malformations and strikes approximately $10-15 \%$ in the general population, with more cases of cavernous sinus and transverse sinus, and rare occurrence of anterior cranial fossa DAVF because of complexity in vascular architecture and hemodynamics of the anterior cranial fossa $[1,2]$, which is more difficult to treat and cure,

\footnotetext{
Manuscript accepted for publication August 7, 2013

${ }^{a}$ Department of Neurosurgery, Fuzhou General Hospital, Fuzhou 350025, China

${ }^{\mathrm{b}}$ Corresponding author: Zheng Liu, Department of Neurosurgery, Fuzhou General Hospital, 156 Second West Ring Road North, Fuzhou 350025, China. Email: liuzhengfz18@126.com
}

doi: http://dx.doi.org/10.4021/jnr230e especially when an aneurysmal dilatation is formed. Here we report three cases of patients with the malformation, hospitalized at our institution during a period from Dec 2011 to Sep 2012, and review possible pathogenesis, diagnosis and treatment for the malformation.

\section{Case Report}

\section{Case 1}

A 58-year-old man was referred to our hospital with a complaint of paroxysmal dizziness and nausea for more than six months. Head CT and MRI examinations found a roundshaped lesion at right anterior cranial fossa and a nearby old hemorrhage (Fig. 1A). Emergency craniotomy was performed and after cleaning of the hemorrhage the lesion was found at the right side of the anterior cranial fossa, with a size of $3 \times 2 \times 2 \mathrm{~cm}$ and a purple membrane (Fig. 1B). A significantly thickening vein on the surface of the lesion was also found, which suddenly ruptured with a large amount of bleeding during operation. The surgeon managed stopping the bleeding and found no connection of it with anterior cerebral arteries. At the bottom of the dura, a feeding artery was found. After clipping of the artery the volume of the lesion decreased significantly and its body was removed. Postoperative DSA showed the DVAF artery stump (Fig. 1C, arrow) and the patient was 8-day postoperatively discharged.

\section{Case 2}

A 56-year-old man presented to our hospital with a complaint of a sudden headache for 2 days. Cranial CT and MRI found a hemorrhage at the end of the left frontal and an aneurysmal dilatation at the bottom of the frontal lobe, which was connected to an abnormal vein that merged to the left sphenoparietal sinus (Fig. 2A, B). During surgical operation the hemorrhage was cleaned and the aneurysmal dilatation with approximately $2 \times 2 \times 1 \mathrm{~cm}$ in size was found. Next to the comb-dural junction, 2 feeding arteries appeared originated from the dura mater and were clipped (Fig. 2C). In addition, a left sphenoparietal sinus drainage vein near the aneurysmal 


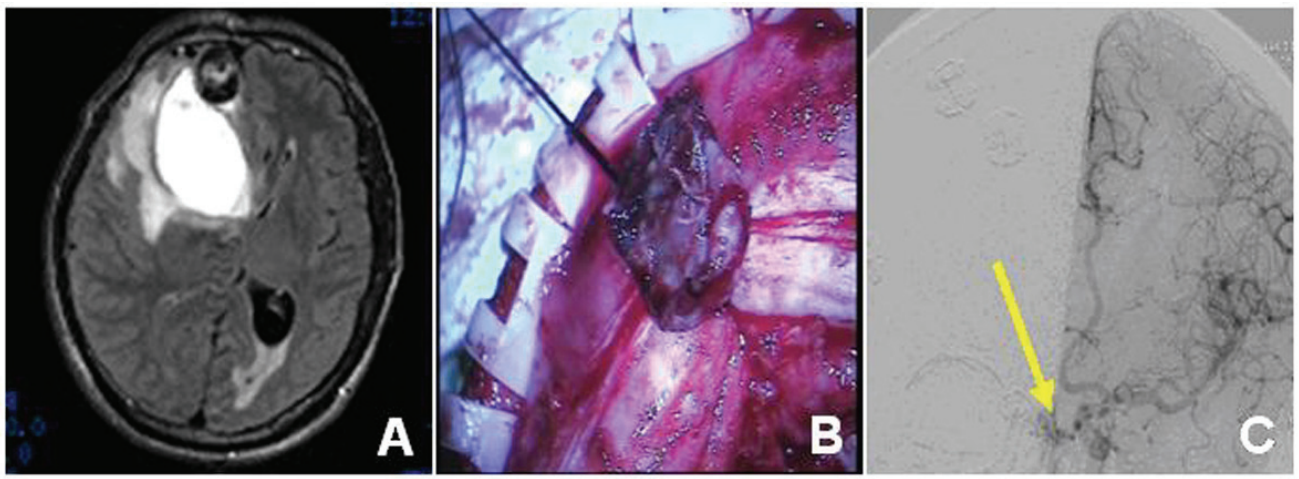

Figure 1. Imaging angiogram and intraoperative findings of case 1 patient. The preoperative CT (A) showed the right frontal lobe blood vessels and the nearby old hemorrhage. The intraoperative findings $(B)$ revealed the aneurysmal dilatation with the purple membrane and the postoperative DSA showed the arteriovenous fistula artery stump (C, arrow).

dilatation was found and its rear part as well as the aneurysmal dilatation was removed. The patient recovered and was discharged 12-day postoperatively.

\section{Case 3}

A 61-year-old man was hospitalized due to a sudden headache for $2 \mathrm{~h}$. Head CT showed subarachnoid hemorrhage at cerebral base cistern (Fig. 3A) and CTA found a thickening of an abnormal blood vessel at the bottom of the right side of the anterior cranial fossa. At its rear end an aneurysmlike malformation was found and a surgery was considered. However, family members of the patient strongly preferred conservative treatment. After 13 days of the treatment the patient showed improvement and was discharged. However, only after 1 day, the patient collapsed at home, was admitted to the hospital immediately. Head CT showed brain hemorrhages at the right frontal lobe and bilateral lateral (Fig. 3B). An emergency craniotomy was performed and severe brain swelling was seen. Next to the comb there existed an abnormal blood vessel near the anterior cranial fossa dura and at its rear blind end an aneurysmal dilatation with a size of $8 \times$
$8 \times 8 \mathrm{~mm}$ was found, still bleeding (Fig. 3C). After occlusion of the feeding vessel the bleeding stopped and the aneurysmal dilatation was removed. The patient had been in coma since surgery and died 14 days postoperatively because of respiratory and circulatory failure.

\section{Discussion}

The anterior cranial fossa DAVF malformation accounts for only approximately $5.8 \%$ of all DAVF cases. The male/female ratio is about 7:1 and $70 \%$ of patients are over 50 years old with a higher bleeding rate, approximately $62-91 \%$ [3]. The pathogenesis of the malformation is still unknown and it has been suggested that it may be related to congenitally abnormal development of anterior cranial fossa vascular and venous sinus [4]. Many lines of evidence have shown that there is a correlation between the formation of DAVF and some related high-risk factors, such as venous sinus thrombosis, inflammation, traumatism, and most importantly, venous sinus hypertension, which plays a decisive role for the malformation $[5,6]$. Since there is no venous sinus at the

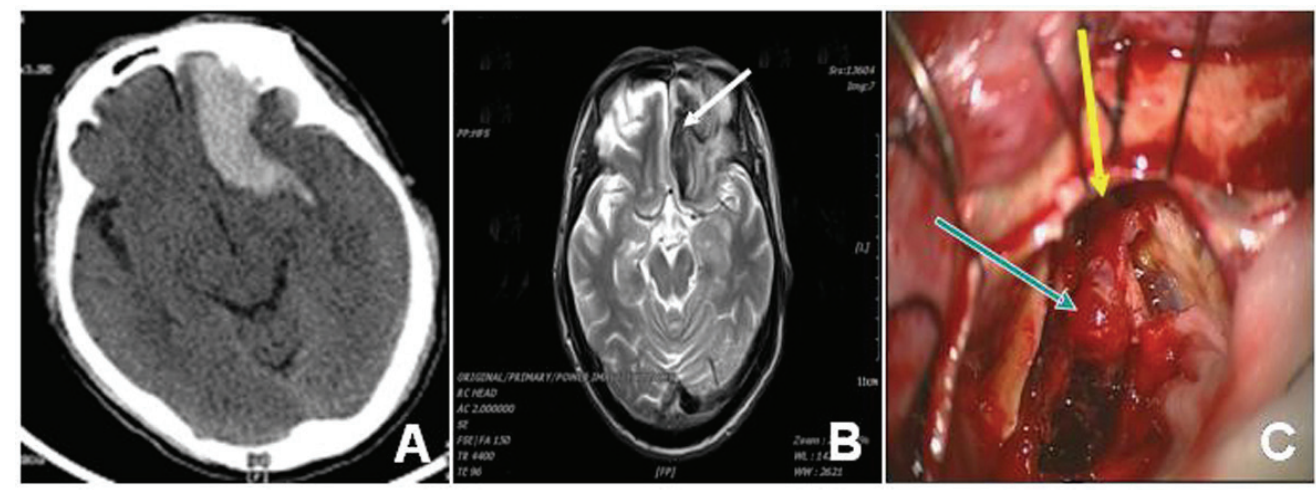

Figure 2. Imaging angiogram and intraoperative findings of case 2 patient. The preoperative CT (A) showed left frontal hemorrhage and the MRI T2-weighted image revealed hypointensity in the drainage vein (B, arrow). The intraoperative findings revealed the aneurysmal dilatation $(\mathrm{C}$, arrow in blue) and its feeding artery ( $\mathrm{C}$, arrow in yellow). 


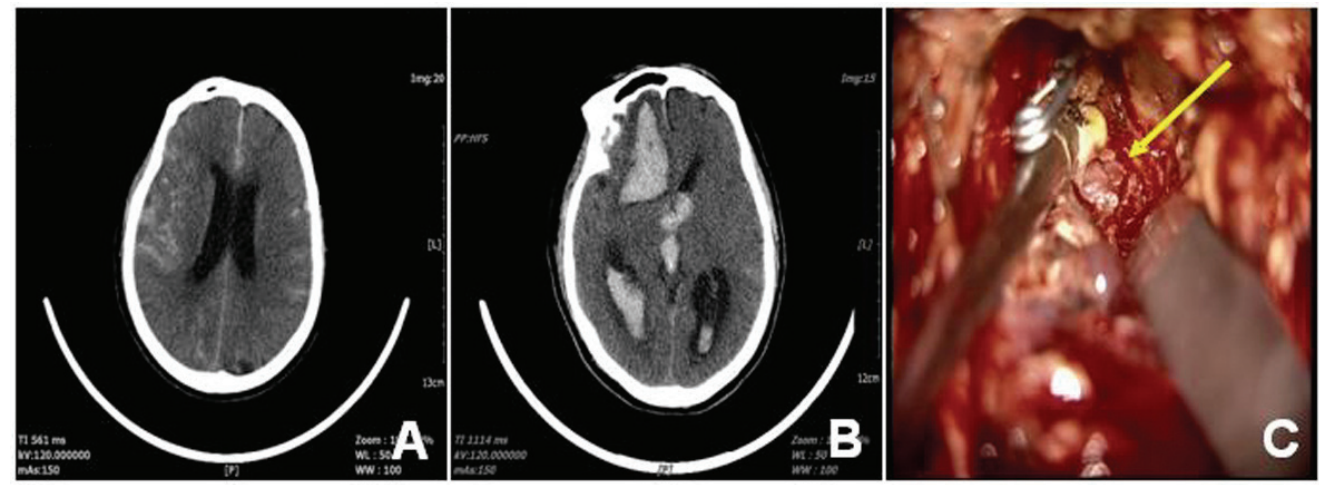

Figure 3. Imaging angiogram and intraoperative findings of case 3 patient. The original CT (A) showed SAH at cerebral base cistern and the preoperative CT (B) after re-admission revealed the hemorrhage in the brain ventricle. The intraoperative findings showed the aneurysmal dilatation (C, arrow).

bottom of anterior cranial fossa, DAVF has a unique and complex angio-architecture structure in this section. Feeding vessels mainly come from skull base anterior ethmoidal arteries, anterior cerebral artery olfactory branches and posterior ethmoidal arteries. The outside middle meningeal artery anterior branches and superficial temporal arteries have also participated in blood supply [7]. The continuing flow of arterial blood into cortical veins has caused constant high-pressure stress in the veins. In addition, the proximal ends of the vein walls become more tortuous, even gradually thinning, and forming venous lake or pathological arterial aneurysmlike structure. It has been reported that $50 \%$ of the anterior cranial fossa DAVF were associated with the "venous lake" or "aneurysmal dilatation", usually occurred in the vicinity of venous channel where the dura mater and pia mater are anastomosed [8]. All three cases reported in this study are in line with the pathological changes.

Rapidly progressive clinical courses, causing severe damage or even death, can be avoided if the anterior cranial fossa DAVF malformation was detected at early stage and treated by surgery or intervention therapies timely. Due to large amounts of arterial blood rapidly flow into nearby sinuses, MRI T1- and T2-weighted images in frontal tortuous can be acquired; sometimes existence of the aneurysmal dilatations can also be detected. Therefore, brain MRI is very important for early detection of the malformation. Furthermore, there is no doubt that DSA is still the gold standard for diagnosis of the malformation and it greatly helps understanding of complex angio-architecture profile of the anterior cranial fossa DAVF.

Because of high re-bleeding risk of the malformation the conservative treatment is not considered a preferred option. Therefore, it was really a pity that the third patient died of rebleeding of the aneurysmal dilatation and delayed surgical treatment. Craniotomy is considered the simplest and most effective method for the treatment of the rare anterior cranial fossa DAVF malformation at present and the surgical occlusion of the fistula in a timely manner is a key to achieve a favorable outcome for patients suffered from the malformation, with a help from intervention and radiation therapies.

\section{Disclosure}

On behalf of all authors, the corresponding author states that there is no conflict of interest.

\section{References}

1. Im SH, Oh CW, Han DH. Surgical management of an unruptured dural arteriovenous fistula of the anterior cranial fossa: natural history for 7 years. Surg Neurol. 2004;62(1):72-75; discussion 75.

2. Tanei T, Fukui K, Wakabayashi K, Mitsui Y, Inoue N, Watanabe M. Dural arteriovenous fistula in the anterior cranial fossa: four case reports. Neurol Med Chir (Tokyo). 2008;48(12):560-563.

3. Geibprasert S, Pereira V, Krings T, Jiarakongmun $P$, Toulgoat F, Pongpech S, Lasjaunias P. Dural arteriovenous shunts: a new classification of craniospinal epidural venous anatomical bases and clinical correlations. Stroke. 2008;39(10):2783-2794.

4. de Haan TR, Padberg RD, Hagebeuk EE, Aronica E, van Rijn RR, Majoie CB, Kok JH. A case of neonatal dural sinus malformation: clinical symptoms, imaging and neuropathological investigations. Eur J Paediatr Neurol. 2008;12(1):41-45.

5. Tirakotai W, Bian LG, Bertalanffy H, Siegfried B, Sure U. Immunohistochemical study in dural arteriovenous fistula and possible role of ephrin-B2 for development of dural arteriovenous fistula. Chin Med J (Engl). 2004;117(12):1815-1820.

6. Bhatia R, Tripathi M, Srivastava A, Garg A, Singh MB, Nanda A, Padma MV, et al. Idiopathic hypertrophic cranial pachymeningitis and dural sinus occlusion: 
two patients with long-term follow up. J Clin Neurosci. 2009;16(7):937-942.

7. Lucas Cde P, Caldas JG, Prandini MN. Do leptomeningeal venous drainage and dysplastic venous dilation predict hemorrhage in dural arteriovenous fistula? Surg Neurol. 2006;66(Suppl 3):S2-5; discussion S5-6.
8. Jamous MA, Satoh K, Satomi J, Matsubara S, Nakajima N, Uno M, Nagahiro S. Detection of enlarged cortical vein by magnetic resonance imaging contributes to early diagnosis and better outcome for patients with anterior cranial fossa dural arteriovenous fistula. Neurol Med Chir (Tokyo). 2004;44(10):516-520; discussion 520-521. 